\title{
Review
}

\section{Plasticity of the human motor cortex and recovery from stroke}

\author{
Mark Hallett* \\ Human Motor Control Section, NINDS, NIH, Medical Neurology Branch, Building 10, Room 5N226, 10 Center Drive MSC 1428, Bethesda, MD \\ 20892-1428, USA
}

\begin{abstract}
By a variety of mechanisms, the human brain is constantly undergoing plastic changes. Plasticity can be studied with phenomena such as peripheral deafferentation and motor learning. Spontaneous recovery from stroke in the chronic stage likely comes about because of plasticity, and the best recovery seems to result from reorganization in the damaged hemisphere. Knowledge about the physiology of brain plasticity has led to the development of new techniques for rehabilitation. Published by Elsevier Science B.V.
\end{abstract}

Theme: Development and regeneration

Topic: Motor systems

Keywords: Plasticity; Rehabilitation; Transcranial magnetic stimulation; Stroke; Amputation; Motor learning; Deafferentation

\section{Contents}

1. Introduction .....

2. Mechanisms of plasticity...

3. Peripheral deafferentation

4. Motor learning

5. Stroke.

6. Stroke rehabilitation.

\section{Introduction}

Stroke is a common and often devastating disorder leading to substantial disability. There are methods of prevention and much current excitement about new techniques for acute intervention in order to limit the amount of brain damage. After the first few hours from stroke onset, however, what damage will be done has been done, and it is time to consider rehabilitation. Fortunately, there is a certain amount of spontaneous recovery. Patients may be given formal physical therapy and occupational therapy, but there has been only little evidence that such methods are actually valuable in promoting neurological improve-

*Tel.: +1-301-496-1561; fax: +1-301-402-1007.

E-mail address: hallettm@ninds.nih.gov (M. Hallett). ment. It is necessary to understand how spontaneous recovery takes place and how it can be facilitated for optimal improvement. Some spontaneous recovery is no doubt due to resolution of edema and possibly recovery of tissue function in tissues that were ischemic but not destroyed. This aspect of recovery is likely to occur within a few days. Another aspect, responsible for recovery beyond this acute period, is likely brain plasticity.

While up to about a decade ago, it was commonly taught that the adult brain was not plastic, it has now been determined that not only is the brain capable of plastic changes, but is constantly changing. A simple observation makes this obvious. Plasticity must be responsible for learning since learning implies a change of the brain. As long as the brain can learn, it can change, and similar changes are likely to be helpful in recovery from injury. 
This review focuses on plastic changes that can be demonstrated in the motor system and, in particular, how such changes appear relevant in recovery from hemiplegia.

\section{Mechanisms of plasticity}

The cellular mechanisms that are likely to be responsible for plasticity in humans are under active investigation. Their physiology has been studied in model systems, and we have only incomplete knowledge so far as to which mechanism applies to which phenomenon. First, a change in the balance of excitation and inhibition can happen very quickly. This process depends on the fact that neurons or neural pathways have a much larger region of anatomical connectivity than their usual territory of functional influence. Some zones may be kept in check by tonic inhibition. If the inhibition is removed, the region of influence can be quickly increased or unmasked [35]; in a process often called unmasking. A second process that can also be relatively fast is strengthening or weakening of existing synapses, in processes such as long-term potentiation (LTP) or long-term depression (LTD) [32,33]. A third process is a change in neuronal membrane excitability [27]. A fourth process is anatomical changes, which need a longer period of time. Specific anatomical changes include sprouting of new axon terminals and formation of new synapses [60]. These processes operate in different time periods and are not mutually exclusive; indeed, one can be followed by another serially and this is likely to occur.

\section{Peripheral deafferentation}

The situation of peripheral deafferentation has been well studied and is briefly introduced here to illustrate some features of human brain plasticity. Merzenich and colleagues [43,44] and Donoghue and colleagues [18,19], working with primates, demonstrated that after deafferentation of a limb there was reorganization of the sensory and motor cortices, respectively. The deafferented (or deefferented) cortex did not stay idle, but was taken over by body representations adjacent to the deafferented body part. This situation can be investigated in the motor system of humans [23-26]. Cohen et al. [13] studied patients with traumatic, surgical or congenital amputations of the arm at about the level of the elbow using transcranial magnetic stimulation (TMS). Motor representation areas targeting muscles ipsilateral and immediately proximal to the stump were larger than those for muscles contralateral to the stump. These results are consistent with the idea that the motor cortex for the muscles proximal to the amputation had expanded into the territory of the amputated part. TMS does confound motor cortex excitability and size of the representation area, but by analogy to the primates, size is likely to have increased.
In order to see how fast modulation of motor outputs can occur in humans, studies can be done with reversible deafferentation of a limb segment. Reversible deafferentation was accomplished by using a blood pressure cuff, at first with, and later without regional local anesthesia [3,4]. The amplitudes of motor evoked potentials (MEPs) to TMS from muscles immediately proximal to the temporarily anesthetized forearm increased in minutes after the onset of anesthesia and returned to control values after the anesthesia subsided. On the other hand, other processes take a much longer time. In patients with brachial plexus avulsion, after the intercostal nerve was anastomosed to the musculocutaneous nerve, Mano et al. [42] showed that projections from the biceps region of the motor cortex can be directed to the spinal cord neurons of the intercostal nerve. This process took a year or more to occur. Thus, some changes occur rapidly and others slowly, suggestive that there are multiple types of phenomena that play a role.

\section{Motor learning}

Cortical changes result from changes in the patterns of behavior, and this fact will be very important in consideration of physical therapy. In order to investigate this issue in humans, Pascual-Leone et al. [51] performed detailed mapping of the motor cortical areas targeting the first dorsal interosseus (FDI) and the adductor digiti minimi (ADM) bilaterally in Braille readers and blind controls with focal TMS. In the controls, motor representations of right and left FDI and ADM were not significantly different. However, in the proficient Braille readers, the representation of the FDI in the reading hand was significantly larger than that in the nonreading hand or in either hand of the controls. Conversely, the representation of the ADM in the reading hand was significantly smaller than that in the non-reading hand or in either hand of the controls. These results suggests that the cortical representation for the reading finger in proficient Braille readers is enlarged at the expense of the representation of other fingers.

Conversely, Liepert et al. [40] studied cortical plasticity in patients who had a unilateral immobilization of the ankle joint without any peripheral nerve lesion. The motor cortex area of the inactivated tibial anterior muscle diminished compared to the unaffected leg without changes in spinal excitability or motor threshold. Representation areas increase or decrease depending on use.

To determine how fast such changes can occur, motor learning can be investigated. Pascual-Leone et al. [52] looked at the motor cortical representation of the hand over a 5-day period in normal subjects as they learned a skilled task with their hand. As subjects became more skilled at a five-finger exercise on a piano, the size (or excitability) of the motor representation of hand increased. Such changes appeared to depend on skill learning since simple use of 
the hand did not lead to similar changes. A similar result has been obtained in several other learning experiments [12] and in a primate model [54].

\section{Stroke}

One theme for stroke recovery has been recruitment of ipsilateral pathways. Strong evidence for this possibility comes from evaluation of patients who have undergone hemispherectomy. After hemispherectomy motor function in the limb contralateral to the excised hemisphere experiences a substantial degree of recovery, particularly when surgery is performed at early age. To understand the mechanisms underlying this recovery of function, Cohen et al. [14] studied patients with hemispherectomy. TMS of the remaining hemisphere induced bilateral activation of deltoid and biceps. Similar findings were obtained by Benecke et al. [1]. Evaluation of MEPs indicated that muscles ipsilateral to the preserved hemisphere were activated by stimulation of scalp positions anterior and lateral to those activating muscles on the normal side. Similarly, ipsilateral elbow movements were associated with regional cerebral blood flow (rCBF) increases with PET in an area centered slightly anterior and lateral to that activated by the same movements on the normal side. These results indicate that ipsilateral and contralateral representations in the remaining hemisphere are topographically differentiated, with ipsilateral representations having a more anterior and lateral scalp distribution. The anatomy suggests that the normal ipsilateral representation has become more influential in these patients and has likely contributed to the recovery.

One of the first suggestions that ipsilateral pathways may also be important in recovery from human stroke came from neuroimaging studies. Chollet et al. [10] studied six patients with hemiplegic stroke from capsular infarction who had recovered full strength, although three of the six had a little residual clumsiness and some had mirror movements of the good hand when they moved the recovered hand. In this group analysis, the recovered hand, compared with the normal hand, showed increased activation of ipsilateral sensorimotor cortex, insula and inferior parietal cortex and contralateral cerebellum. Subsequently, others have pointed out the prominence of ipsilateral activation in stroke recovery $[5,16,34,65]$.

Studies with TMS have not confirmed the utility of ipsilateral pathways for recovery when stroke occurs in adult age. Some studies do show that ipsilateral MEPs are more likely, have lower threshold and have shorter latency in patients with stroke than normal subjects $[6,36,62,63]$. However, these responses are found more frequently in patients with poorer functional recovery. Turton et al. [62] studied 21 patients within 5 weeks of the onset of a stroke and then at regular intervals over the next 12 months and found that the presence of contralateral MEPs at the start of the study was a good indicator of recovery. In addition, contralateral MEPs appeared at or just before the time of recovery of hand movement in those who recovered it later during the study. They also found nine subjects with ipsilateral MEPs in affected hand muscles, evoked by stimulation of the intact hemisphere, most of whom had poor recovery of hand function. These ipsilateral MEPs were generally of low amplitude. It may well be that there is competition between contralateral and ipsilateral connections, and that the poorly functioning ipsilateral pathways are more prominent when the functional contralateral pathways cannot recover.

The ipsilateral pathways may be more functional in children with prenatal or perinatal lesions, since the earlier the damage, the more likely the quality of the improvement [7,41]. If the lesion is prenatal, the ipsilateral corticospinal tract may even have individual axons with branches that supply homologous motor neuron pools on the two sides [7]. On the other hand, in the study of Carr et al. [7], the best function was seen in patients with MEPs to stimulation of the cortex contralateral to the hemiplegia. Ipsilateral MEPs were found in subjects with both good and poor function of the affected hand. When contralateral MEPs were not present, hand function was poor unless intense mirroring was present. Therefore, ipsilateral MEPs by themselves are not evidence of functionally significant ipsilateral control even in children.

The one situation where ipsilateral pathways are likely important is in recovery from dysphagia. While swallowing problems can affect as many as one in three patients in the period immediately after a stroke, it usually recovers completely within weeks. This impressive propensity for recovery is likely to relate to how the area of the motor cortex concerned with swallowing is organized and then reorganized after cerebral injury as demonstrated by Hamdy et al. [30]. Their studies have indicated that swallowing has a bilateral but asymmetric hemispheric representation. Damage to the hemisphere that has the greater swallowing output appears to predispose that individual to swallowing problems, while damage to the hemisphere with the smaller swallowing output will not affect swallowing [28]. However, when there is dysphagia, because there is additional substrate for swallowing in the undamaged hemisphere, the capacity for compensatory reorganization in the contralateral motor cortex can be increased, leading to a greater likelihood of recovery. This has been demonstrated in a study of 28 patients who had a unilateral hemispheric stroke [29]. Dysphagia was initially present in $71 \%$ of patients and in 46 and $41 \%$ of the patients at 1 and 3 months, respectively. Nondysphagic and persistently dysphagic patients showed little change in pharyngeal representation in either hemisphere at 1 and 3 months compared with presentation, but dysphagic patients who recovered had an increased pharyngeal representation in the unaffected hemisphere at 1 and 3 months without change in the affected hemisphere. Hence, return of 
swallowing is associated with increased pharyngeal representation in the unaffected hemisphere, indicating a critical role for the intact hemisphere reorganization in recovery.

The intact hemisphere also clearly plays a role in recovery of other midline muscles, such as the tongue. Muellbacher et al. [47] used TMS of the motor cortex to study motor reorganization for control of the tongue after stroke. Six patients presented with a unilateral lingual paralysis after limited monohemispheric ischemia. The first examination was performed during the symptomatic stage and was repeated after complete recovery of lingual function had been established. In control subjects TMS of either hemisphere invariably produced contralateral and ipsilateral compound muscle action potentials (CMAPs). In most individuals an asymmetric cortical motor output pattern was found, as significantly larger CMAPs were recorded from the contralateral lingual muscles than from the ipsilateral muscles. During the symptomatic stage, in the six patients with a unilateral lingual paralysis a similar pattern was found by stimulating the intact hemisphere, whereas TMS of the affected hemisphere failed to elicit a CMAP on either side. At the time of recovery, only one patient showed evidence of complete recovery of the primarily affected hemisphere. In the remaining five patients, the unilateral interruption of the corticonuclear pathways persisted in spite of complete functional recovery implying that the recovery of lingual movements must be attributed to the intact hemisphere.

Plastic changes in the damaged hemisphere are likely generally most efficient in producing the best recovery. Most TMS studies have shown that presence of contralateral MEPs early after the stroke is a marker for good recovery $[2,8,9,20,31,46,53,55,62]$. Preservation of corticospinal tract with MRI imaging was also found to correlate with good recovery confirming the TMS studies [2]. Clearly, contralateral control, even if reorganized, seems superior to ipsilateral control in recovery. Reorganization of motor cortex after lesioning can occur, as demonstrated by Nudo and colleagues [49,50] in the primate, but this appears to require attempted use of the weakened body part. With recovery following stroke, Rossini and co-workers [11,56,61] have demonstrated enlarged and/or relocated TMS maps of the recovering muscles, indicating the probable importance of contralateral hemisphere plasticity mechanisms in recovery. Weiller et al. [66] looked at the individual patterns of cerebral activation in eight patients with good stroke recovery compared with the pattern of a group of ten normal subjects. They found a large ventral extension of the hand field of the contralateral sensorimotor cortex in all patients with lesions of the posterior limb of the internal capsule. They also found a greater activation than normal in variable combinations of the supplementary motor areas, the insula, the frontal operculum, and the parietal cortex. They also saw that structures belonging to motor pathways ipsilateral to the recovered limb were more activated in the patients than in normal subjects. However, additional activation of the ipsilateral sensorimotor cortex was only found in the four patients who exhibited associated movements of the unaffected hand when the recovered hand performed the motor task. All these data confirm that contralateral plasticity is better than ipsilateral plasticity in producing good improvement.

TMS studies can be useful for more than mapping. Leipert et al. [39] have demonstrated decreased inhibition in patients with acute stroke. This may come about for two reasons. GABA neurons may be more sensitive to ischemia. On the other hand, plasticity is enhanced with diminished inhibition, and this may be a sign of the cortex's attempt to repair itself.

\section{Stroke rehabilitation}

Using principles of plasticity, it should be possible to improve on spontaneous recovery. One simple point is that use of a limb is critical for achieving and maintaining cortical representation. Often in physical therapy, emphasis has been on accomplishing tasks of daily living and not on improving function of the weakened limb. If the weakened limb is not exercised, then, insult can be added to injury, and the limb representational area will be further limited by its disuse. Taub and colleagues $[45,58,59]$ have used this principle to suggest what is called constraint-induced movement therapy. This method forces use of the hemiplegic limb by contraining the good limb. In a number of clinical trials, there have been behavioral improvement even in patients with chronic and apparently stable stroke. Liepert and co-workers [38,39] have shown that TMS maps increase in size in this circumstance showing that the expected cortical changes appear to be occurring.

Another method for improving rehabilitation is the combined use of drug therapy together with the physical therapy. Pharmacological intervention can alter plastic changes. The best documented influence is with amphetamine and related noradrenergic agents [21,22]. First demonstrated to be valuable in a rat model, there are now several clinical trials showing that amphetamine given together with physical therapy is better than physical therapy alone [17,64]. The mechanism for this drug action is not completely clear. While it is possible that it has effect by relieving diaschisis, amphetamine also enhances plastic changes in motor learning situations in both animals and humans [37,57].

We are currently investigating a new method that takes advantage of several features of plasticity. After hemiplegic stroke, there is often significant loss of hand function with relatively retained strength in the proximal arm muscles [15]. Since there is a 'competition' between body parts for territory in the motor cortex, it is possible that use of the proximal muscles makes it difficult for hand muscles to increase their representation. We know that peripheral 
deafferentation with a tourniquet increases the MEP of proximal muscles, and recently we have demonstrated that this increase is magnified by exercise of the proximal muscles during the peripheral block [67]. We reasoned that anesthesia of proximal muscles and exercise of the hand might increase cortical representation of the hand and concomitantly improve hand function. Preliminary investigation suggests that this may indeed occur [48]. Thus, we can use knowledge of the physiology of plasticity to create new therapies for chronic stroke victims.

\section{References}

[1] R. Benecke, B.U. Meyer, H.-J. Freund, Reorganization of descending motor pathways in patients after hemispherectomy and severe hemispheric lesions demonstrated by magnetic brain stimulation, Exp. Brain Res. 83 (1991) 419-426.

[2] F. Binkofski, R.J. Seitz, S. Arnold, J. Classen, R. Benecke, H.J. Freund, Thalamic metabolism and corticospinal tract integrity determine motor recovery in stroke, Ann. Neurol. 39 (1996) 460470.

[3] J.P. Brasil-Neto, L.G. Cohen, A. Pascual-Leone, F.K. Jabir, R.T. Wall, M. Hallett, Rapid reversible modulation of human motor outputs after transient deafferentation of the forearm: A study with transcranial magnetic stimulation, Neurology 42 (1992) 1302-1306.

[4] J.P. Brasil-Neto, J. Valls-Solé, A. Pascual-Leone, A. Cammarota, V.E. Amassian, R. Cracco, P. Maccabee, J. Cracco, M. Hallett, L.G. Cohen, Rapid modulation of human cortical motor outputs following ischemic nerve block, Brain 116 (1993) 511-525.

[5] Y. Cao, L. D'Olhaberriague, E.M. Vikingstad, S.R. Levine, K.M. Welch, Pilot study of functional MRI to assess cerebral activation of motor function after poststroke hemiparesis, Stroke 29 (1998) 112122.

[6] M.D. Caramia, C. Iani, G. Bernardi, Cerebral plasticity after stroke as revealed by ipsilateral responses to magnetic stimulation, NeuroReport 7 (1996) 1756-1760.

[7] L.J. Carr, L.M. Harrison, A.L. Evans, J.A. Stephens, Patterns of central motor reorganization in hemiplegic cerebral palsy, Brain 116 (1993) 1223-1247.

[8] A. Catano, M. Houa, J.M. Caroyer, H. Ducarne, P. Noel, Magnetic transcranial stimulation in acute stroke: early excitation threshold and functional prognosis, Electroencephalogr. Clin. Neurophysiol. 101 (1996) 233-239.

[9] A. Catano, M. Houa, J.M. Caroyer, H. Ducarne, P. Noel, Magnetic transcranial stimulation in non-haemorrhagic sylvian strokes: interest of facilitation for early functional prognosis, Electroencephalogr. Clin. Neurophysiol. 97 (1995) 349-354.

[10] F. Chollet, V. DiPiero, R.J.S. Wise, D.J. Brooks, R.J. Dolan, R.S.J. Frackowiak, The functional anatomy of motor recovery after stroke in humans. A study with positron emission tomography, Ann. Neurol. 29 (1991) 63-71.

[11] P. Cicinelli, R. Traversa, P.M. Rossini, Post-stroke reorganization of brain motor output to the hand: a 2-4 month follow-up with focal magnetic transcranial stimulation, Electroencephalogr. Clin. Neurophysiol. 105 (1997) 438-450.

[12] J. Classen, J. Liepert, S.P. Wise, M. Hallett, L.G. Cohen, Rapid plasticity of human cortical movement representation induced by practice, J. Neurophysiol. 79 (1998) 1117-1123.

[13] L.G. Cohen, S. Bandinelli, T.W. Findley, M. Hallett, Motor reorganization after upper limb amputation in man, Brain 114 (1991) $615-627$.

[14] L.G. Cohen, B.J. Roth, E.M. Wassermann, H. Topka, P. Fuhr, J. Schultz, M. Hallett, Magnetic stimulation of the human cerebral cortex, an indicator of reorganization in motor pathways in certain pathological conditions, J. Clin. Neurophysiol. 8 (1991) 56-65.

[15] J.G. Colebatch, S.C. Gandevia, The distribution of muscular weakness in upper motor neuron lesions affecting the arm, Brain 112 (1989) 749-763.

[16] S.C. Cramer, G. Nelles, R.R. Benson, J.D. Kaplan, R.A. Parker, K.K. Kwong, D.N. Kennedy, S.P. Finklestein, B.R. Rosen, A functional MRI study of subjects recovered from hemiparetic stroke, Stroke 28 (1997) 2518-2527.

[17] E.A. Crisostomo, P.W. Duncan, M. Propst, D.V. Dawson, J.N. Davis, Evidence that amphetamine with physical therapy promotes recovery of motor function in stroke patients, Ann. Neurol. 23 (1988) 94-97.

[18] J.P. Donoghue, Plasticity of adult sensorimotor representations, Curr. Opin. Neurobiol. 5 (1995) 749-754.

[19] J.P. Donoghue, S. Suner, J.N. Sanes, Dynamic organization of primary motor cortex output to target muscles in adult rats. II. Rapid reorganization following motor nerve lesions, Exp. Brain Res. 79 (1990) 492-503.

[20] J.V. Escudero, J. Sancho, D. Bautista, M. Escudero, J. Lopez-Trigo, Prognostic value of motor evoked potential obtained by transcranial magnetic brain stimulation in motor function recovery in patients with acute ischemic stroke, Stroke 29 (1998) 1854-1859.

[21] D.M. Feeney, From laboratory to clinic: noradrenergic enhancement of physical therapy for stroke or trauma patients, Adv. Neurol. 73 (1997) 383-394.

[22] D.M. Feeney, M.P. Weisend, A.E. Kline, Noradrenergic pharmacotherapy, intracerebral infusion and adrenal transplantation promote functional recovery after cortical damage, J. Neural Transplant. Plast. 4 (1993) 199-213.

[23] M. Hallett, Motor cortex plasticity, in: G. Comi, C.H. Lücking, J. Kimura, P.M. Rossini (Eds.), Clinical Neurophysiology: From Receptors to Perception, Vol. 50, Elsevier, Amsterdam, 1999, pp. 85-91.

[24] M. Hallett, Plasticity, in: J.C. Mazziotta, A.W. Toga, R.S.J. Frackowiak (Eds.), Brain Mapping; The Disorders, Academic Press, San Diego, 2000, pp. 569-586, Chapter 23.

[25] M. Hallett, Plasticity in the human motor system, Neuroscientist 5 (1999) 324-332.

[26] M. Hallett, L.G. Cohen, A. Pascual-Leone, J. Brasil-Neto, E.M. Wassermann, A.N. Cammarota, Plasticity of the human motor cortex, in: A.F. Thilmann, D.J. Burke, W.Z. Rymer (Eds.), Spasticity: Mechanisms and Management, Springer, Berlin, 1993, pp. 6781.

[27] J.A. Halter, J.S. Carp, J.R. Wolpaw, Operantly conditioned motoneuron plasticity: possible role of sodium channels, J. Neurophysiol. 73 (1995) 867-871.

[28] S. Hamdy, Q. Aziz, J.C. Rothwell, R. Crone, D. Hughes, R.C. Tallis, D.G. Thompson, Explaining oropharyngeal dysphagia after unilateral hemispheric stroke, Lancet 350 (1997) 686-692.

[29] S. Hamdy, Q. Aziz, J.C. Rothwell, M. Power, K.D. Singh, D.A. Nicholson, R.C. Tallis, D.G. Thompson, Recovery of swallowing after dysphagic stroke relates to functional reorganization in the intact motor cortex, Gastroenterology 115 (1998) 1104-1112.

[30] S. Hamdy, J.C. Rothwell, Gut feelings about recovery after stroke: the organization and reorganization of human swallowing motor cortex, Trends Neurosci. 21 (1998) 278-282.

[31] A. Heald, D. Bates, N.E. Cartlidge, J.M. French, S. Miller, Longitudinal study of central motor conduction time following stroke. 2 . Central motor conduction measured within $72 \mathrm{~h}$ after stroke as a predictor of functional outcome at 12 months, Brain 116 (1993) $1371-1385$.

[32] G. Hess, C.D. Aizenman, J.P. Donoghue, Conditions for the induction of long-term potentiation in layer II/III horizontal connections of the rat motor cortex, J. Neurophysiol. 75 (1996) 17651778.

[33] G. Hess, J.P. Donoghue, Long-term depression of horizontal connections in rat motor cortex, Eur. J. Neurosci. 8 (1996) 658-665. 
[34] M. Honda, T. Nagamine, H. Fukuyama, Y. Yonekura, J. Kimura, H. Shibasaki, Movement-related cortical potentials and regional cerebral blood flow change in patients with stroke after motor recovery, J. Neurol. Sci. 146 (1997) 117-126.

[35] K.M. Jacobs, J.P. Donoghue, Reshaping the cortical motor map by unmasking latent intracortical connections, Science 251 (1991) 944947.

[36] T. Lammers, J. Netz, V. Hömberg, Disinhibition of ipsilateral MEPresponses in stroke patients (abstract), Electroencephalogr. Clin. Neurophysiol. 97 (1995) S193-S194.

[37] E.H. Lee, Y.L. Ma, Amphetamine enhances memory retention and facilitates norepinephrine release from the hippocampus in rats, Brain Res. Bull. 37 (1995) 411-416.

[38] J. Liepert, W.H. Miltner, H. Bauder, M. Sommer, C. Dettmers, E. Taub, C. Weiller, Motor cortex plasticity during constraint-induced movement therapy in stroke patients, Neurosci. Lett. 250 (1998) $5-8$.

[39] J. Liepert, P. Storch, A. Fritsch, C. Weiller, Motor cortex disinhibition in acute stroke, Clin. Neurophysiol. 111 (2000) 671-676.

[40] J. Liepert, M. Tegenthoff, J.P. Malin, Changes of cortical motor area size during immobilization, Electroencephalogr. Clin. Neurophysiol. 97 (1995) 382-386.

[41] Y. Maegaki, Y. Maeoka, K. Takeshita, Plasticity of central motor pathways in hemiplegic children with large hemispheric lesions (abstract), Electroencephalogr. Clin. Neurophysiol. 97 (1995) S192.

[42] Y. Mano, T. Nakamuro, R. Tamura, T. Takayanagi, K. Kawanishi, S. Tamai, R.F. Mayer, Central motor reorganization after anastomosis of the musculocutaneous and intercostal nerves in patients with traumatic cervical root avulsion, Ann. Neurol. 38 (1995) $15-20$.

[43] M.M. Merzenich, W.M. Jenkins, Reorganization of cortical representations of the hand following alterations of skin inputs induced by nerve injury, skin island transfers, and experience, J. Hand Ther. 6 (1993) 89-104.

[44] M.M. Merzenich, R.J. Nelson, M.P. Stryker, M.S. Cynader, A. Schoppmann, J.M. Zook, Somatosensory cortical map changes following digit amputation in adult monkeys, J. Comp. Neurol. 224 (1984) 591-605.

[45] W.H. Miltner, H. Bauder, M. Sommer, C. Dettmers, E. Taub, Effects of constraint-induced movement therapy on patients with chronic motor deficits after stroke: a replication, Stroke 30 (1999) 586-592.

[46] U.K. Misra, J. Kalita, Motor evoked potential changes in ischaemic stroke depend on stroke location, J. Neurol. Sci. 134 (1995) 67-72.

[47] W. Muellbacher, C. Artner, B. Mamoli, The role of the intact hemisphere in recovery of midline muscles after recent monohemispheric stroke, J. Neurol. 246 (1999) 250-256.

[48] W. Muellbacher, C. Richards, U. Ziemann, G. Wittenberg, D. Weltz, B. Boroojerdi, L.G. Cohen, M. Hallett, Improving hand function in chronic stroke, (2001) submitted for publication.

[49] R.J. Nudo, G.W. Milliken, Reorganization of movement representations in primary motor cortex following focal ischemic infarcts in adult squirrel monkeys, J. Neurophysiol. 75 (1996) 2144-2149.

[50] R.J. Nudo, B.M. Wise, F. SiFuentes, G.W. Milliken, Neural substrates for the effects of rehabilitative training on motor recovery after ischemic infarct, Science 272 (1996) 1791-1794.

[51] A. Pascual-Leone, A. Cammarota, E.M. Wassermann, J.P. BrasilNeto, L.G. Cohen, M. Hallett, Modulation of motor cortical outputs to the reading hand of Braille readers, Ann. Neurol. 34 (1993) $33-37$.
[52] A. Pascual-Leone, N. Dang, L.G. Cohen, J.P. Brasil-Neto, A. Cammarota, M. Hallett, Modulation of muscle responses evoked by transcranial magnetic stimulation during the acquisition of new fine motor skills, J. Neurophysiol. 74 (1995) 1037-1045.

[53] G. Pennisi, G. Rapisarda, R. Bella, V. Calabrese, A. Maertens De Noordhout, P.J. Delwaide, Absence of response to early transcranial magnetic stimulation in ischemic stroke patients: prognostic value for hand motor recovery, Stroke 30 (1999) 2666-2670.

[54] E.J. Plautz, G.W. Milliken, R.J. Nudo, Effects of repetitive motor training on movement representations in adult squirrel monkeys: role of use versus learning, Neurobiol. Learn. Mem. 74 (2000) $27-55$.

[55] G. Rapisarda, E. Bastings, A. Maertens de Noordhout, G. Pennisi, P.J. Delwaide, Can motor recovery in stroke patients be predicted by early transcranial magnetic stimulation?, Stroke 27 (1996) 21912196.

[56] P.M. Rossini, C. Caltagirone, A. Castriota-Scanderbeg, P. Cicinelli, C. Del Gratta, M. Demartin, V. Pizzella, R. Traversa, G.L. Romani, Hand motor cortical area reorganization in stroke: a study with fMRI, MEG and TCS maps, NeuroReport 9 (1998) 2141-2146.

[57] E. Soetens, S. Casaer, R. Dn'Hooge, J.E. Hueting, Effect of amphetamine on long-term retention of verbal material, Psychopharmacology (Berl.) 119 (1995) 155-162.

[58] E. Taub, N.E. Miller, T.A. Novack, E.W.d. Cook, W.C. Fleming, C.S. Nepomuceno, J.S. Connell, J.E. Crago, Technique to improve chronic motor deficit after stroke, Arch. Phys. Med. Rehabil. 74 (1993) 347-354.

[59] E. Taub, G. Uswatte, R. Pidikiti, Constraint-induced movement therapy: a new family of techniques with broad application to physical rehabilitation: a clinical review, J. Rehabil. Res. Dev. 36 (1999) 237-251.

[60] N. Toni, P.A. Buchs, I. Nikonenko, C.R. Bron, D. Muller, LTP promotes formation of multiple spine synapses between a single axon terminal and a dendrite, Nature 402 (1999) 421-425.

[61] R. Traversa, P. Cicinelli, A. Bassi, P.M. Rossini, G. Bernardi, Mapping of motor cortical reorganization after stroke. A brain stimulation study with focal magnetic pulses, Stroke 28 (1997) 110-117.

[62] A. Turton, S. Wroe, N. Trepte, C. Fraser, R.N. Lemon, Contralateral and ipsilateral EMG responses to transcranial magnetic stimulation during recovery of arm and hand function after stroke, Electroencephalogr. Clin. Neurophysiol. 101 (1996) 316-328.

[63] A. Turton, S. Wroe, N. Trepti, C. Fraser, R.N. Lemon, Ipsilateral EMG responses to transcranial magnetic stimulation during recovery of arm and hand function after stroke (abstract), Electroencephalogr. Clin. Neurophysiol. 97 (1995) S192.

[64] D. Walker-Batson, P. Smith, S. Curtis, H. Unwin, R. Greenlee, Amphetamine paired with physical therapy accelerates motor recovery after stroke. Further evidence, Stroke 26 (1995) 2254-2259.

[65] C. Weiller, F. Chollet, K.J. Friston, R.J.S. Wise, R.S.J. Frackowiak, Functional reorganization of the brain in recovery from striatocapsular infarction in man, Ann. Neurol. 31 (1992) 463-472.

[66] C. Weiller, S.C. Ramsay, R.J. Wise, K.J. Friston, R.S.J. Frackowiak, Individual patterns of functional reorganization in the human cerebral cortex after capsular infarction, Ann. Neurol. 33 (1993) 181-189.

[67] U. Ziemann, W. Muellbacher, M. Hallett, L.G. Cohen, Modulation of practice-dependent plasticity in human motor cortex, Brain (2001) in press. 